\title{
EGFR Mutation Status in Lung Adenocarcinoma-Associated Malignant Pleural Effusion and Efficacy of EGFR Tyrosine Kinase Inhibitors
}

\author{
Jiyoul Yang, $M D^{1}$ \\ Ok-Jun Lee, MD, $\mathrm{PhD} 2,3$ \\ Seung-Myoung Son, MD2 \\ Chang Gok Woo, MD2 \\ Yusook Jeong, MD \\ Yaewon Yang, MD \\ Jihyun Kwon, $M D^{1}$ \\ Ki Hyeong Lee, MD, $\mathrm{PhD}^{1,4}$ \\ Hye Sook Han, MD, PhD 1.4
}

Departments of ${ }^{1}$ Internal Medicine and ${ }^{2}$ Pathology, Chungbuk National University Hospital, Cheongju, Departments of ${ }^{3}$ Pathology and ${ }^{4}$ Internal Medicine, Chungbuk National University College of Medicine, Cheongju, Korea

\begin{abstract}
Purpose
Malignant pleural effusions (MPEs) are often observed in lung cancer, particularly adenocarcinoma. The aim of this study was to investigate epidermal growth factor receptor (EGFR) mutation status in lung adenocarcinoma-associated MPEs (LA-MPEs) and its correlation with efficacy of EGFR tyrosine kinase inhibitor (TKI) therapy.
\end{abstract}

\section{Materials and Methods}

Samples comprised 40 cell blocks of pathologically-confirmed LA-MPEs collected before the start of EGFR TKI therapy. EGFR mutation status was re-evaluated by peptide nucleic acid clamping and the clinical outcomes of EGFR TKI-treated patients were analyzed retrospectively.

\section{Results}

EGFR mutations were detected in $72.5 \%$ of LA-MPE cell blocks (29/40). The median progression-free survival for patients with EGFR mutations in LA-MPEs was better than that for patients with wild-type EGFR (7.33 months vs. 2.07 months; hazard ratio, 0.486; 95\% confidence interval, 0.206 to $1.144 ; p=0.032$ ). The objective response rate (ORR) of 26 patients with EGFR mutations in LA-MPEs among the 36 patients with measurable lesions was $80.8 \%$, while the ORR of the 10 patients with wild-type EGFR in LA-MPEs was $10 \%$ $(p<0.001)$. Among the 26 patients with EGFR mutations in LA-MPEs, the ORR of target lesions and LA-MPEs were $88.5 \%$ and $61.5 \%$, respectively $(p=0.026)$.

\section{Conclusion}

EGFR mutation status in cell blocks of LA-MPEs confirmed by pathologic diagnosis is highly predictive of EGFR TKI efficacy. For patients with EGFR mutations in LA-MPEs, the response to EGFR TKIs seems to be worse for pleural effusions than for solid tumors.
Correspondence: Hye Sook Han, MD, PhD Department of Internal Medicine, Chungbuk National University College of Medicine,

1 Chungdae-ro, Seowon-gu, Cheongju 28644, Korea Tel: 82-43-269-6306

Fax: 82-43-273-3252

E-mail: sook3529@hanmail.net

Received August 13, 2017

Accepted September 14, 2017

Published Online September 19, 2017
Key words

Epidermal growth factor receptor, Lung adenocarcinoma, Pleural effusion, Tyrosine kinase inhibitor

\section{Introduction}

Lung cancer is a leading cause of cancer-related mortality worldwide, with non-small cell lung cancer (NSCLC) accounting for around $80 \%-85 \%$ of lung cancers [1]. Most NSCLC patients are diagnosed at an advanced stage and less than $30 \%$ of patients undergo surgical resection [2]. The initial therapy for advanced NSCLC is systemic chemotherapy with platinum-based doublets, but the response is poor (median overall survival, approximately 1 year) [3]. By contrast, treatment with epidermal growth factor receptor (EGFR) tyrosine kinase inhibitors (TKIs) leads to a significant improvement in the prognosis of patients with advanced NSCLC, particularly those harboring EGFR gene mutations [4]. These mutations comprise small in-frame deletions and substitutions clustered around the ATP-binding site in exons $18,19,20$, and 21 of EGFR; these mutations increase the affinity of ATPase and EGFR TKIs [5,6]. EGFR mutations occur in the majority of female, non-smoking Asian patients with 
lung adenocarcinoma [5,6]. Successful treatment of advanced lung adenocarcinoma with EGFR TKIs means that EGFR mutation status can be used to select patients who will benefit from treatment with these drugs. Currently, tumor tissue obtained at surgery or biopsy are used for EGFR mutation tests [7]; unfortunately, it is difficult to obtain an adequate amount of tumor tissue from patients with advanced NSCLC. Moreover, the often poor performance status of patients with advanced NSCLC means that they may not tolerate diagnostic procedures or the location of the tumor may make it impossible to obtain tissue. Thus, new types of sample are needed to replace or supplement current samples to detect EGFR mutations.

Malignant pleural effusion (MPE) is present in approximately $50 \%$ of advanced NSCLC patients, most frequently in those with lung adenocarcinoma [8,9]. This adenocarcinoma grows in the peripheral lung tissue and invades the pleural cavity easily. Sampling pleural effusion is a relatively simple, non-invasive, and repeatable procedure, and MPE is a feasible source of tumor DNA for molecular analysis [9]. Therefore, MPE samples are useful when no tumor tissues are available for mutation analysis. Although many studies show that MPEs from NSCLC patients are a good material in which to detect EGFR mutations [10-19], there are insufficient data to evaluate the utility of MPEs for determining overall EGFR mutation rates and the efficacy of EGFR TKIs.

Here, we selected patients using pathologically-confirmed lung adenocarcinoma-associated MPE (LA-MPE) samples and re-evaluated EGFR mutation status using peptide nucleic acid (PNA) clamping, which has a sensitivity of $1 \%$. The clinical outcome of patients receiving EGFR TKIs was then analyzed retrospectively. Tumor response to EGFR TKI therapy was analyzed by examining the response of target lesions and pleural effusions separately.

\section{Materials and Methods}

\section{Patients and sample collection}

From March 2010 to August 2016, cases from Chungbuk National University Hospital were reviewed using a pathologic database of lung adenocarcinoma patients that contained cell blocks of MPEs. All MPEs excluded tuberculosis pleurisy through acid-fast bacilli staining and tuberculosispolymerase chain reaction (PCR). All cell blocks of MPE samples contained adenocarcinoma cells. Ethanol-fixed, paraffinembedded cell blocks of MPEs obtained from histologicallyor cytologically-confirmed lung adenocarcinoma patients who showed no evidence of another malignant tumor were included. Lung adenocarcinoma was confirmed by pathology reports of primary tumor biopsies or of cell blocks of MPEs that stained positive for thyroid transcription factor1. Matched formalin-fixed, paraffin-embedded surgically resected or biopsy specimens of primary lung adenocarcinoma were also retrieved for comparison. All cell blocks of LA-MPEs or primary lung adenocarcinoma tissues were collected before the start of EGFR TKI therapy. Clinical characteristics, including age, sex, smoking history, and EGFR TKI therapy, were obtained from medical records. EGFR TKIs were divided into three groups according to the initial EGFR TKI agent used: (1) gefitinib or erlotinib were classified as first generation EGFR TKIs; (2) afatinib or dacomitinib were classified as second generation EGFR TKIs; and (3) osimertinib, rociletinib, and HM61713 were classified as third generation EGFR TKIs.

\section{DNA extraction and PNA clamping}

DNA was extracted from five representative paraffin sections $(10 \mu \mathrm{m})$ cut from cell blocks and tumor tissue. Prior to DNA isolation, the tissue was deparaffinized in xylene and then washed in $70 \%$ ethanol. DNA was isolated using a High Pure PCR Template Preparation Kit (Roche Applied Science, Mannheim, Germany), according to the manufacturer's protocol. The DNA was eluted in $50 \mu \mathrm{L}$ elution buffer, and the concentration and purity were assessed using a NanoDrop ND-1000 spectrophotometer (NanoDrop Technologies, Wilmington, DE). The extracted DNA was stored at $-20^{\circ} \mathrm{C}$ until required.

A PNAClamp EGFR Mutation Detection Kit (PANAGENE, Inc., Daejeon, Korea) was used to detect EGFR mutations by real-time PCR (RT-PCR), as previously described $[17,18]$. The assay, based on PNA clamping technology, quickly and accurately detects specific mutations or deletions at known positions within the EGFR gene. Detection of 47 mutations in exons 18, 19, 20, and 21 of EGFR is possible. Briefly, all reactions were carried out in a volume of $20 \mu \mathrm{L}$ containing template DNA, a primer, a PNA probe set, and SYBR Green PCR master mix. RT-PCR was performed using a CFX 96 apparatus (Bio-Rad, Philadelphia, PA). The thermocycling conditions were as follows: 5 minutes at $94^{\circ} \mathrm{C}$, followed by 40 cycles at $94^{\circ} \mathrm{C}$ for 30 seconds, $70^{\circ} \mathrm{C}$ for 20 seconds, $63^{\circ} \mathrm{C}$ for 30 seconds, and $72^{\circ} \mathrm{C}$ for 30 seconds. PCR efficiency was determined by evaluating threshold cycle $(\mathrm{Ct})$ values, which were calculated automatically from PCR amplification plots of SYBR Green fluorescence versus number of cycles. Delta $\mathrm{Ct}(\Delta \mathrm{Ct})$ was calculated as the $\mathrm{Ct}$ value of the standard $\mathrm{Ct}$ minus the $\mathrm{Ct}$ value of the sample. Higher $\Delta \mathrm{Ct}$ values indicate more efficient amplification of the mutant. A cutoff value of 2.0 was used to indicate the presence of mutant DNA. 


\section{Efficacy of EGFR TKI therapy}

All patients were treated with EGFR TKI therapy and the response was evaluated, although some were defined as mutation-negative. Progression-free survival (PFS) was calculated from the start of EGFR TKI therapy until confirmed disease progression or death. Based on serial imaging studies, treatment response was classified as follows: complete response (CR), partial response (PR), stable disease (SD), or progressive disease (PD) according to the Response Evaluation Criteria in Solid Tumors 1.1 criteria [20]. Objective response rate (ORR) was defined as the percentage of patients with a CR or PR. The size of the pleural effusion was defined as follows: Massive, effusion volume $>75 \%$ of the hemithorax; large, effusion volume $50 \%-75 \%$ of the hemithorax; moderate, effusion volume $25 \%-50 \%$ of the hemithorax; and small, effusion volume $<25 \%$ of the hemithorax. Pleural effusion responses were evaluated by computed tomography before and after EGFR TKI therapy. Modified pleural effusion response criteria from the Japan Lung Cancer Society were adopted [21-23]: CR was defined as complete disappearance of pleural fluid lasting for at least 4 weeks; PR was defined as a distinguishable decrease (at least a 30\% decrease in the volume of pleural effusion) for more than 4 weeks; PD was defined as appreciable progression of effusion (at least a 20\% increase in the volume of pleural effusion) or need of drainage within 4 weeks; and SD was defined as failure to meet the above criteria or no change in the amount of pleural effusion, which required no thoracentesis for more than 4 weeks. Effusion response rate was defined as the percentage of patients with a CR or PR.

\section{Statistical analysis}

Categorical variables were analyzed using Pearson's chisquare test (except where a small size required the use of Fisher exact test). PFS after EGFR TKI therapy was analyzed by the Kaplan-Meier method, and groups were compared using the log-rank test. All statistical tests were two-sided and $\mathrm{p}$-values $<0.05$ were defined as statistically significant. All statistical analyses were performed using SPSS for Windows software, ver. 18.0 (SPSS Inc., Chicago, IL).

\section{Ethical statement}

The study, including the retrospective use of archival cell blocks of LA-MPEs or tissue for EGFR mutation analysis, was reviewed and approved by the Institutional Review Board of Chungbuk National University Hospital.

Written informed consents were obtained; however, the informed consents were waived in patients who died at the time of the study.

\section{Results}

\section{Patient characteristics}

Of the 204 cell blocks of LA-MPEs confirmed by pathologic diagnosis that were screened between March 2010 and August 2016, 40 that were collected before the start of EGFR
Lung adenocarcinoma associated MPEs confirmed by pathologic diagnosis $(n=204)$

\section{Did receive EGFG TKI therapy $(n=152)$}

Lung adenocarcinoma associated MPEs collected after the start of EGFR TKI therapy ( $n=9$ ) Died before the evaluation of response to EGFR TKI (n=3)
Lung adenocarcinoma associated MPEs collected before the start of EGFR TKI therapy $(n=40)$

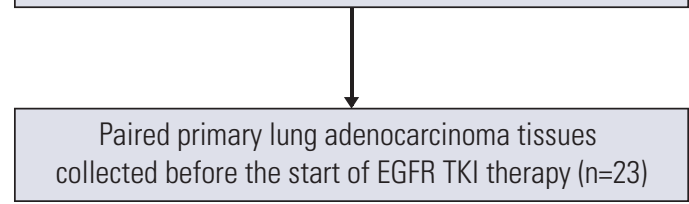

Fig. 1. Flow diagram. MPE, malignant pleural effusion; EGFR TKI, epidermal growth factor receptor tyrosine kinase inhibitor. 
Table 1. Patient characteristics

\begin{tabular}{|c|c|}
\hline Characteristic & No. of patients $(n=40)$ \\
\hline \multicolumn{2}{|l|}{ Age at diagnosis (yr) } \\
\hline Median & $71(42-94)$ \\
\hline \multicolumn{2}{|l|}{ Sex } \\
\hline Male & $15(37.5)$ \\
\hline Female & $25(62.5)$ \\
\hline \multicolumn{2}{|l|}{ ECOG performance status } \\
\hline 0 & $3(7.5)$ \\
\hline 1 & $36(90.0)$ \\
\hline 2 & $1(2.5)$ \\
\hline \multicolumn{2}{|l|}{ Smoking history } \\
\hline Never smoker & $25(62.5)$ \\
\hline Former and current smoker & $11(27.5)$ \\
\hline Unknown & $4(10.0)$ \\
\hline \multicolumn{2}{|l|}{ Size of the pleural effusion } \\
\hline Small & $13(32.5)$ \\
\hline Moderate & $11(27.5)$ \\
\hline Large & $8(20.0)$ \\
\hline Massive & $8(20.0)$ \\
\hline \multicolumn{2}{|l|}{ EGFR TKI therapy } \\
\hline 1st line & $27(67.5)$ \\
\hline 2nd line & $11(27.5)$ \\
\hline$\geq 3$ rd line & $2(5.0)$ \\
\hline \multicolumn{2}{|l|}{ Generation EGFR TKI } \\
\hline 1 st & $34(85.0)$ \\
\hline 2nd & $4(10.0)$ \\
\hline 3rd & $2(5.0)$ \\
\hline
\end{tabular}

ECOG, Eastern Cooperative Oncology Group; EGFR TKI, epidermal growth factor receptor tyrosine kinase inhibitor.

TKI therapy were analyzed (Fig. 1). Among them, 23 patients had paired surgically resected or biopsy specimens of primary lung adenocarcinoma. The clinical characteristics of the 40 patients are listed in Table 1 . The median age was 71 years (range, 42 to 94 years). Twenty-five patients $(62.5 \%)$ were female and $25(62.5 \%)$ were never-smokers. The patients were grouped according to the size of the pleural effusion: 13 patients $(32.5 \%)$ had a small effusion, $11(27.5 \%)$ had a moderate effusion, and eight patients $(20.0 \%)$ each had large and massive effusions. The initial EGFR TKI was administered as a first-line treatment during the course of the disease in $27(67.5 \%)$, as a second-line treatment in $11(27.5 \%)$, and as a third-line or further treatment in two $(5.0 \%)$. Thirty-four patients $(85 \%)$ were treated with first generation EGFR TKIs such as gefitinib or erlotinib.

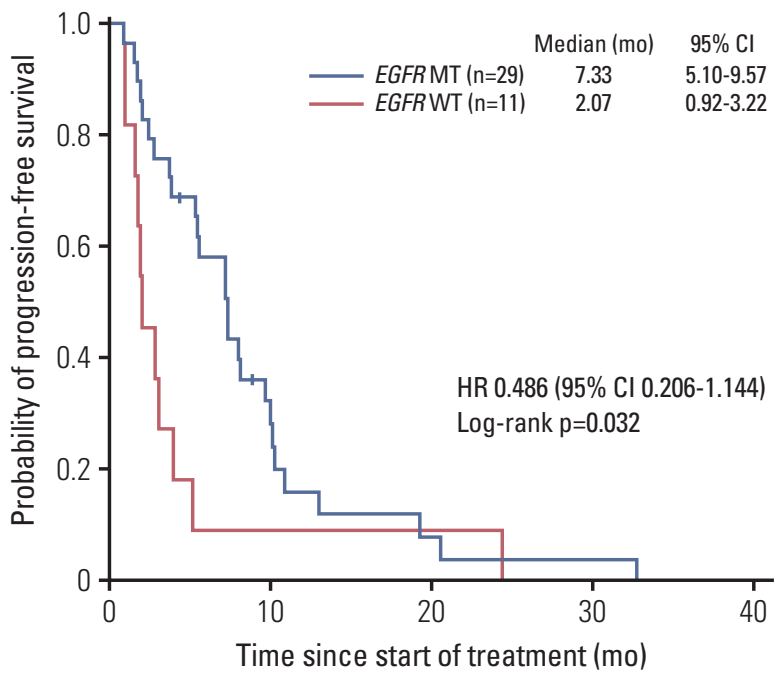

Fig. 2. Progression-free survival according to EGFR mutation status in cell blocks of malignant pleural effusion. EGFR, epidermal growth factor receptor; WT, wildtype; MT, mutant-type; CI, confidence interval; HR, hazard ratio.

\section{EGFR mutation status in LA-MPEs and the efficacy of EGFR TKI therapy}

The median PFS for patients with EGFR mutations in LA-MPEs was significantly longer than that for patients with wild-type EGFR (7.33 vs. 2.07 months; hazard ratio, 0.486; 95\% confidence interval, 0.206 to 1.144; $p=0.032$ ) (Fig. 2).

The tumor response to EGFR TKIs among the 36 patients with measurable lesions according to EGFR mutation status in LA-MPEs is shown in Table 2. Of the 26 patients with EGFR mutations in LA-MPE, $21(80.8 \%)$ showed a PR, one $(3.8 \%)$ showed SD, and four $(15.4 \%)$ showed PD. Of the 10 patients with wild-type EGFR in LA-MPEs, one (10.0\%) showed a PR, four $(40.0 \%)$ showed SD, and five $(50.0 \%)$ showed PD. EGFR mutation status in LA-MPEs correlated with the overall tumor response to EGFR TKI therapy $(\mathrm{p}<0.001)$.

The tumor response of target lesions and pleural effusions to EGFR TKIs among the 26 patients with measurable lesions and EGFR mutations in LA-MPEs is shown in Table 3. Tumor response of target lesions showed PR of $88.5 \%$, SD of $3.8 \%$, and PD of $7.7 \%$. Tumor response of pleural effusions showed CR of $15.4 \%$, PR of $46.1 \%$, SD of $11.5 \%$, and PD of $26.5 \%$. Among the 26 patients with EGFR mutations in LA-MPEs, the ORR of the target lesions and LA-MPEs were $88.5 \%$ and $61.5 \%$, respectively $(\mathrm{p}=0.026)$. 
Table 2. Tumor response to EGFR tyrosine kinase inhibitors

\begin{tabular}{lccc} 
Response & Total $(\mathrm{n}=36)$ & \multicolumn{2}{c}{ EGFR mutation status of LA-MPEs } \\
\cline { 3 - 4 } & & & \\
Best overall response & $22(61.1)$ & $21(80.8)$ & $1(10.0)$ \\
PR & $5(13.9)$ & $1(3.8)$ & $4(40.0)$ \\
SD & $9(25.0)$ & $4(15.4)$ & $5(50.0)$ \\
PD & EGFR WT (n=10) & $<0.001$ \\
\hline
\end{tabular}

Values are presented as number (\%). EGFR, epidermal growth factor receptor; LA-MPE, lung adenocarcinoma-associated malignant pleural effusion; MT, mutant type; WT, wild type; PR, partial response; SD, stable disease; PD, progressive disease.

Table 3. Comparison of the response to EGFR tyrosine kinase inhibitors between target lesions and pleural effusions in patients with EGFR MT-MPE

\begin{tabular}{lccc} 
& \multicolumn{2}{c}{ EGFR MT in LA-MPE (n=26) } & p-value \\
\cline { 2 - 2 } Best overall response & Target lesions & Pleural effusion & \\
CR & & & \\
PR & 0 & $(15.4)$ & 0.007 \\
SD & $23(88.5)$ & $3(46.2)$ & \\
PD & $1(3.8)$ & $7(26.9)$ & 0.026 \\
\hline Objective response & $2(7.7)$ & $16(61.5)$ & 0 \\
\hline
\end{tabular}

Values are presented as number (\%). EGFR, epidermal growth factor receptor; MPE, malignant pleural effusion; MT, mutant type; LA-MPE, lung adenocarcinoma-associated MPE; CR, complete response; PR, partial response; SD, stable disease; PD, progressive disease.

Table 4. EGFR mutation status in malignant pleural effusion and primary lung adenocarcinoma

\begin{tabular}{lcc} 
EGFR mutation status & $\begin{array}{c}\text { EGFR mutation status } \\
\text { of LA-MPEs (n=40) }\end{array}$ & $\begin{array}{c}\text { EGFR mutation status of } \\
\text { primary lung adenocarcinoma }(\mathrm{n}=\mathbf{2 3})\end{array}$ \\
\hline EGFR WT & $11(27.5)$ & $4(17.4)$ \\
EGFR MT & $29(72.5)$ & $19(82.6)$ \\
Exon 19 deletion & $17(58.6)$ & $12(63.2)$ \\
Exon 21 L858R & $9(31.0)$ & $5(26.3)$ \\
Exon 19 deletion and exon 21 L858R & $2(6.9)$ & $2(10.5)$ \\
\hline Exon 21 L861R & $1(3.4)$ & 0 \\
\hline
\end{tabular}

EGFR, epidermal growth factor receptor; LA-MPE, lung adenocarcinoma-associated malignant pleural effusion; WT, wild type; MT, mutant type.

\section{1) EGFR mutation status in LA-MPEs and corresponding primary lung adenocarcinomas}

EGFR mutations were found in 29/40 LA-MPEs and 19/23 primary lung adenocarcinomas $(72.5 \%$ vs. $82.6 \%)$. The dif- ference was not statistically significant $(\mathrm{p}=0.060)$ (Table 4$)$. The most frequent mutations observed in LA-MPEs and primary lung adenocarcinomas were a deletion mutation in exon 19 and a point mutation (L858R) in exon 21. EGFR mutations in primary lung adenocarcinomas and LA-MPEs 
Table 5. Comparison of EGFR mutation status among patients with malignant pleural effusions and primary lung adenocarcinomas

\begin{tabular}{|c|c|c|c|c|c|c|c|}
\hline \multirow[b]{2}{*}{ Case } & \multicolumn{3}{|c|}{ Patient characteristic } & \multicolumn{2}{|c|}{ EGFR mutation status } & \multicolumn{2}{|c|}{ Response } \\
\hline & Sex & Age (yr) & Smoking & $\begin{array}{c}\text { Primary lung } \\
\text { adenocarcinoma }\end{array}$ & LA-MPEs & Target lesions & Pleural effusion \\
\hline 1 & $\mathrm{~F}$ & 71 & Never & L858R & L858R & PR & $\mathrm{CR}$ \\
\hline 2 & $\mathrm{M}$ & 42 & Current & Exon 19 del & Exon 19 del & PR & PR \\
\hline 3 & $\mathrm{M}$ & 81 & Former & Exon 19 del & Exon 19 del & PR & PR \\
\hline 4 & $\mathrm{~F}$ & 59 & Never & Exon 19 del & Exon 19 del & PR & PR \\
\hline 5 & $\mathrm{~F}$ & 77 & Never & Exon 19 del & Exon 19 del & PR & PR \\
\hline 6 & $\mathrm{M}$ & 81 & Current & Exon 19 del & Exon 19 del & No & PR \\
\hline 7 & $\mathrm{~F}$ & 52 & Never & L858R & Exon 19 del/L858R & PR & SD \\
\hline 8 & $\mathrm{~F}$ & 46 & Never & Exon 19 del & Exon 19 del & No & SD \\
\hline 9 & $\mathrm{~F}$ & 66 & Never & L858R & L858R & PR & PD \\
\hline 10 & M & 87 & Former & Exon $19 \mathrm{del} / \mathrm{L} 858 \mathrm{R}$ & L858R & PR & PD \\
\hline 11 & M & 82 & Former & Exon 19 del/L858R & Exon 19 del & PR & PD \\
\hline 12 & $\mathrm{M}$ & 58 & Former & Exon 19 del & Exon 19 del & PR & PD \\
\hline 13 & $\mathrm{~F}$ & 68 & Never & Exon 19 del & Exon 19 del & SD & PD \\
\hline 14 & $\mathrm{~F}$ & 68 & Never & Exon 19 del & Exon 19 del & PD & PD \\
\hline 15 & M & 62 & Former & WT & Exon 19 del/L858R & PR & PD \\
\hline 16 & $\mathrm{~F}$ & 62 & Current & L858R & WT & SD & PR \\
\hline 17 & $\mathrm{~F}$ & 72 & Never & Exon 19 del & WT & PD & PD \\
\hline 18 & M & 71 & Former & Exon 19 del & WT & SD & SD \\
\hline 19 & $\mathrm{~F}$ & 94 & Never & L858R & WT & SD & PD \\
\hline 20 & M & 62 & Never & L858R & WT & SD & PD \\
\hline 21 & M & 71 & Former & WT & WT & SD & PD \\
\hline 22 & $\mathrm{~F}$ & 81 & Never & WT & WT & No & PR \\
\hline 23 & $\mathrm{M}$ & 71 & Current & WT & WT & $\mathrm{PD}$ & $\mathrm{PD}$ \\
\hline
\end{tabular}

EGFR, epidermal growth factor receptor; LA-MPE, lung adenocarcinoma-associated malignant pleural effusion; PR, partial response; $\mathrm{CR}$, complete response; $\mathrm{SD}$, stable disease; $\mathrm{PD}$, progressive disease; $\mathrm{WT}$, wild type.

from the same patients are presented on a patient-by-patient basis in Table 5. EGFR mutation status between primary lung adenocarcinomas and corresponding LA-MPEs showed a discordance rate of $26.1 \%$ ( 6 of 23 patients, case Nos. 15-20). Among the six patients, one harbored an EGFR mutation in the LA-MPE but not in the primary lung adenocarcinoma and five with EGFR mutations in the primary lung adenocarcinoma had no mutations in LA-MPEs. Among the 14 patients with EGFR mutations in both primary lung adenocarcinoma and LA-MPEs (case Nos. 1-14), the pleural effusion in six (case Nos. 9-14) showed PD in response to EGFR TKIs. The target lesions including the primary lung tumor showed a PR, whereas the response of the pleural effusion was classified as PD in the four patients with EGFR mutations in both the primary lung adenocarcinoma and LA-MPEs (case Nos. 9-12).

\section{Discussion}

Here, we found that EGFR mutation status in cell blocks of LA-MPEs confirmed by pathologic diagnosis is highly predictive of EGFR TKI efficacy. Furthermore, patients with advanced lung adenocarcinoma and pleural effusions that harbor EGFR mutations in LA-MPEs respond less well to EGFR TKIs than those with solid tumors.

Advances in technologies that have very high sensitivity for detecting mutations in a few tumor cells show that EGFR mutations are detectable in pleural effusion samples [9-19], and that the EGFR mutation status of a pleural effusion is a useful predictor of the clinical outcome for NSCLC patients treated with EGFR TKIs $[10,11,14-16,18]$. The present study examined only lung adenocarcinoma and cytologically-confirmed MPEs, and analyzed patients that had previously received EGFR TKIs. The results show that the EGFR mutation rate in patients with pleural effusion $(78.5 \%)$ was higher 
than reported previously. Although previous studies demonstrate the benefits of using body fluids to detect EGFR mutations, the association between EGFR mutation status in LA-MPEs and the efficacy of EGFR TKIs has remained relatively unexplored. However, most previous studies included fewer patients showing a response to EGFR TKIs, or used EGFR mutation tests with high false-negative rates or systemic body fluid samples such as plasma samples $[10,11$, 14-16,18]. Liu et al. [14] analyzed the correlation between the response to EGFR TKIs and EGFR mutation status in 32 cellfree pleural fluid samples using the Amplification Refractory Mutation System method; however, the ORR for patients with wild-type EGFR in pleural fluid was $60 \%$, and false-negative results were still detected. Jian et al. [15] used PCR and Taqman-Minor Groove Binding probes to analyze EGFR mutations in 56 plasma and 32 cell-free pleural effusion samples from NSCLC patients and examined the correlation with responses to gefitinib. Response rates in those with mutant EGFR were significantly higher than in those with wild-type EGFR (90.9\% vs. 9.1\%, respectively). Here, we selected only cell block samples of LA-MPEs containing cytologically-confirmed adenocarcinoma cells. The EGFR mutation status in LA-MPEs was then analyzed using PNA clamping, a method with a sensitivity of $1 \%$. Overall, the tumor response was significantly higher $(80.8 \%$ vs. $10.0 \%)$ and PFS (7.33 months vs. 2.07 months) significantly longer in patients with EGFR mutations than in patients with wild-type EGFR in LA-MPEs, respectively. Although previous studies show that when sensitive genotyping assays are used, cell-free pleural fluid provides the same mutational information as pleural effusion cells $[13,19]$, it is impossible to evaluate precisely whether the tumor-derived DNA was adequately contained since extracted free DNA arises not only from tumor cells but also from necrotic or apoptotic non-tumor cells. Furthermore, the fraction of tumor cells in MPE may be very small, which means that any signal generated by somatic mutations may be easily obscured by the background signal generated by wild-type alleles. Therefore, the sensitivity of mutation detection assay is very critical, and false-negative results would become a major problem if an insensitive assay was used. Therefore, if EGFR mutation testing is to be performed in pleural effusion, we recommend that for MPE containing cytologically-confirmed adenocarcinoma cells, cell pellets rather than cell-free fluid is used, and that sensitive methods such as PNA clamping, rather than insensitive methods, are used.

Previous studies discussed the efficacy of EGFR TKIs in EGFR-mutated NSCLC patients with pleural effusion [24]; the results reveal that the response rate was only slightly lower than that observed in previous prospective randomized trials, in which treatment duration with gefitinib was considerably shorter. This is consistent with our findings of similar TKI response rates in patients with EGFR-mutated pleural effusion, although they show a shorter PFS than other patients with EGFR mutation examined in previous studies. Our study also examined responses to EGFR TKI therapy according to the EGFR mutation status of LA-MPEs; this was done by examining the response of target lesions and pleural effusions separately. Among patients with EGFR mutations in LA-MPEs, the response rate of the target lesions and pleural effusions was $88.5 \%$ and $65.4 \%$, respectively. For patients harboring EGFR mutations in LA-MPEs, responses to EGFR TKIs seem to be worse in pleural effusions than in target lesions. This suggests that lung adenocarcinoma patients with MPE may exhibit unique pathophysiology, and that this interferes with EGFR TKI treatment outcome. One hypothesis is that the tissue surrounding the pleural space or physiological third space created by the pleural effusion may be a barrier to effective transport of EGFR TKIs. However, Masago et al. [25] report good penetration of erlotinib into the pleural space, resulting in marked accumulation of erlotinib in MPE upon repeated dosing. MPE is traditionally attributed to occlusion of parietal pleural stromata or obstruction of lymph vessels which is underscored by inflammation, increased angiogenesis and vascular leakage [9]. If obstruction of the pleural fluid flow occurs by destroying the structure of the lymph node in NSCLC patients with MPE, the total volume of pleural fluid may not decrease, even if EGFR TKI therapy is used to kill cancer cells in the pleural fluid. Another hypothesis is that cancer stem cells in the pleural effusion contribute to the high therapeutic failure rates observed in patients with MPE. A recent study proposed that cancer stem cells in the pleural effusion contribute to the metastatic cascade via epithelial-mesenchymal transition (EMT), anoikis, and adaptation to the microenvironment, which may explain the high therapeutic failure rates in patients with MPE [26]. By reducing cell-cell or cell-matrix interactions, cancer cells enter the serous cavities where they become detached from the parent tumor and are rendered "homeless." The floating malignant cells, which are in a state of anoikis in effusions, should succumb to apoptosis and disappear [27]. However, in reality, the opposite occurs because these cells are uniquely capable of proliferating progressively despite the presumably unfavorable microenvironment. The effusions may serve as a reservoir for tumor cells in the pleural cavity, where they can progress through EMT, become invasive again, and establish further metastases, which then function as a dynamic reservoir that can supply the cancer tissue through autocrine and / or paracrine interactions with growth factors (e.g., vascular endothelial growth factor, basic fibroblast growth factor, and transforming growth factor $\beta$ ) and other stimuli such as chemokines and cytokines produced mainly by cellular components, including mesothelial cells, some stromal cells, and inflammatory cells [28-30]. 
MPEs are filled with tumor cells that have the properties of cancer stem cells after interacting with the microenvironment [26]. A growing body of evidence suggests a relationship between EMT, the emergence of cancer stem cells, and drug resistance [30].

The present study has some limitations. First, this study retrospectively analyzed only patients previously exposed to EGFR TKIs to confirm the association between EGFR mutation status in LA-MPEs and efficacy of EGFR TKI therapy; therefore, it is not possible to present the overall EGFR mutation rate in pleural effusion in patients with LA-MPE. Second, we did not analyze the follow-up cytology of LA-MPEs to evaluate negative cytologic findings after EGFR TKI therapy. There are no standard criteria for evaluating the effusion response to treatment in patients with MPEs. Similar to our study, most investigations have attempted to evaluate patients by correlating the treatment response with traditional solid tumor responses without looking at cytologic findings [22,23]. However, according to the response criteria of the Japan Lung Cancer Society, changes in the volume of pleural effusion alone should not be used to assess responses since they do not truly reflect alterations in tumor burden; also, negative cytologic findings are also important for defining the response [21]. Third, therapeutic thoracentesis, chest tube drainage, and intrapleural administration of sclerosing agents or anticancer agents may influence the prediction of response to pleural effusion. In this study, 30 patients (75\%) underwent more than 2 times (more than $1 \mathrm{~L} /$ time) of ther- apeutic thoracentesis or chest tube drainage before tumor response evaluation. The pleural effusion response was lower than the solid tumor response, despite the possibility of being measured accurately by these additional procedures. Finally, we could not analyze the mechanism underlying poor responses of MPE to EGFR TKIs because serial sampling of pleural effusion or analysis of molecular biomarkers unique to the pathophysiology of LA-MPE were not performed.

EGFR mutation status in LA-MPEs is highly predictive of the efficacy of EGFR TKI therapy in patients with lung adenocarcinoma. Pleural effusions in patients harboring EGFR mutations in LA-MPEs do not respond to EGFR TKIs as well as solid tumors. Further research is necessary to elucidate the mechanisms underlying the poor response of pleural effusion to EGFR TKIs and to develop new strategies that extend the benefits of EGFR TKIs and subsequent treatments in patients with LA-MPEs.

\section{Conflicts of Interest}

Conflict of interest relevant to this article was not reported.

\section{Acknowledgments}

This work was supported by the research grant of the Chungbuk National University Hospital in 2016.

\section{References}

1. Siegel RL, Miller KD, Jemal A. Cancer Statistics, 2017. CA Cancer J Clin. 2017;67:7-30.

2. Spiro SG, Silvestri GA. One hundred years of lung cancer. Am J Respir Crit Care Med. 2005;172:523-9.

3. Schiller JH, Harrington D, Belani CP, Langer C, Sandler A, Krook J, et al. Comparison of four chemotherapy regimens for advanced non-small-cell lung cancer. N Engl J Med. 2002;346: 92-8.

4. Tan DS, Yom SS, Tsao MS, Pass HI, Kelly K, Peled N, et al. The International Association for the Study of Lung Cancer consensus statement on optimizing management of EGFR mutation-positive non-small cell lung cancer: status in 2016. J Thorac Oncol. 2016;11:946-63.

5. Lee DH, Srimuninnimit V, Cheng R, Wang X, Orlando M. Epidermal growth factor receptor mutation status in the treatment of non-small sell lung cancer: lessons learned. Cancer Res Treat. 2015;47:549-54.

6. Lynch TJ, Bell DW, Sordella R, Gurubhagavatula S, Okimoto RA, Brannigan BW, et al. Activating mutations in the epider- mal growth factor receptor underlying responsiveness of nonsmall-cell lung cancer to gefitinib. N Engl J Med. 2004;350: 2129-39.

7. Do H, Krypuy M, Mitchell PL, Fox SB, Dobrovic A. High resolution melting analysis for rapid and sensitive EGFR and KRAS mutation detection in formalin fixed paraffin embedded biopsies. BMC Cancer. 2008;8:142.

8. Roberts ME, Neville E, Berrisford RG, Antunes G, Ali NJ; BTS Pleural Disease Guideline Group. Management of a malignant pleural effusion: British Thoracic Society pleural disease guideline 2010. Thorax. 2010;65 Suppl 2:ii32-40.

9. Froudarakis ME. Pleural effusion in lung cancer: more questions than answers. Respiration. 2012;83:367-76.

10. Soh J, Toyooka S, Aoe K, Asano H, Ichihara S, Katayama H, et al. Usefulness of EGFR mutation screening in pleural fluid to predict the clinical outcome of gefitinib treated patients with lung cancer. Int J Cancer. 2006;119:2353-8.

11. Kimura H, Fujiwara Y, Sone T, Kunitoh H, Tamura T, Kasahara $\mathrm{K}$, et al. EGFR mutation status in tumour-derived DNA 
from pleural effusion fluid is a practical basis for predicting the response to gefitinib. Br J Cancer. 2006;95:1390-5.

12. Wu SG, Gow CH, Yu CJ, Chang YL, Yang CH, Hsu YC, et al. Frequent epidermal growth factor receptor gene mutations in malignant pleural effusion of lung adenocarcinoma. Eur Respir J. 2008;32:924-30.

13. Zhang X, Zhao Y, Wang M, Yap WS, Chang AY. Detection and comparison of epidermal growth factor receptor mutations in cells and fluid of malignant pleural effusion in non-small cell lung cancer. Lung Cancer. 2008;60:175-82.

14. Liu Y, Liu B, Li XY, Li JJ, Qin HF, Tang CH, et al. A comparison of ARMS and direct sequencing for EGFR mutation analysis and tyrosine kinase inhibitors treatment prediction in body fluid samples of non-small-cell lung cancer patients. J Exp Clin Cancer Res. 2011;30:111.

15. Jian G, Songwen Z, Ling Z, Qinfang D, Jie Z, Liang T, et al. Prediction of epidermal growth factor receptor mutations in the plasma/ pleural effusion to efficacy of gefitinib treatment in advanced non-small cell lung cancer. J Cancer Res Clin Oncol. 2010;136:1341-7.

16. Han HS, Eom DW, Kim JH, Kim KH, Shin HM, An JY, et al. EGFR mutation status in primary lung adenocarcinomas and corresponding metastatic lesions: discordance in pleural metastases. Clin Lung Cancer. 2011;12:380-6.

17. Han HS, Lim SN, An JY, Lee KM, Choe KH, Lee KH, et al. Detection of EGFR mutation status in lung adenocarcinoma specimens with different proportions of tumor cells using two methods of differential sensitivity. J Thorac Oncol. 2012;7: 355-64.

18. Yeo CD, Kim JW, Kim KH, Ha JH, Rhee CK, Kim SJ, et al. Detection and comparison of EGFR mutations in matched tumor tissues, cell blocks, pleural effusions, and sera from patients with NSCLC with malignant pleural effusion, by PNA clamping and direct sequencing. Lung Cancer. 2013;81: 207-12.

19. Liu D, Lu Y, Hu Z, Wu N, Nie X, Xia Y, et al. Malignant pleural effusion supernatants are substitutes for metastatic pleural tumor tissues in EGFR mutation test in patients with advanced lung adenocarcinoma. PLoS One. 2014;9:e89946.

20. Eisenhauer EA, Therasse P, Bogaerts J, Schwartz LH, Sargent D, Ford R, et al. New response evaluation criteria in solid tumours: revised RECIST guideline (version 1.1). Eur J Cancer. 2009;45:228-47.

21. Japan Lung Cancer Society. Classification of lung cancer. Tokyo: Kanehara \& Co., Ltd.; 2000. p. 49-52.

22. Masago K, Fujimoto D, Fujita S, Hata A, Kaji R, Ohtsuka K, et al. Response to bevacizumab combination chemotherapy of malignant pleural effusions associated with non-squamous non-small-cell lung cancer. Mol Clin Oncol. 2015;3:415-9.

23. Fujita A, Takabatake H, Tagaki S, Sekine K. Combination chemotherapy in patients with malignant pleural effusions from non-small cell lung cancer : cisplatin, ifosfamide, and irinotecan with recombinant human granulocyte colony-stimulating factor support. Chest. 2001;119:340-3.

24. Wang TF, Chu SC, Lee JJ, Yang GG, Huang WH, Chang ET, et al. Presence of pleural effusion is associated with a poor prognosis in patients with epidermal growth factor receptormutated lung cancer receiving tyrosine kinase inhibitors as first-line treatment. Asia Pac J Clin Oncol. 2017;13:304-13.

25. Masago K, Togashi Y, Fukudo M, Terada T, Irisa K, Sakamori $\mathrm{Y}$, et al. Plasma and pleural fluid pharmacokinetics of erlotinib and its active metabolite OSI-420 in patients with non-smallcell lung cancer with pleural effusion. Clin Lung Cancer. 2011;12:307-12.

26. Chen SF, Lin YS, Jao SW, Chang YC, Liu CL, Lin YJ, et al. Pulmonary adenocarcinoma in malignant pleural effusion enriches cancer stem cell properties during metastatic cascade. PLoS One. 2013;8:e54659.

27. Liotta LA, Kohn E. Anoikis: cancer and the homeless cell Nature. 2004;430:973-4.

28. Cheng D, Lee YC, Rogers JT, Perkett EA, Moyers JP, Rodriguez RM, et al. Vascular endothelial growth factor level correlates with transforming growth factor-beta isoform levels in pleural effusions. Chest. 2000;118:1747-53.

29. Sikora JJ, Dworacki GT, Kaczmarek MT, Jenek RE, Zeromski JO. Immunosuppressive mechanisms in the microenvironment of malignant pleural effusions. Cancer Detect Prev. 2004;28:325-30.

30. Singh A, Settleman J. EMT, cancer stem cells and drug resistance: an emerging axis of evil in the war on cancer. Oncogene. 2010:29:4741-51. 